\title{
Surgical treatment of thalamic fractures treated by anatomic plate with survevent
}

\begin{abstract}
Introduction: Calcaneus fractures are frequent and serious injuries. They represent $65 \%$ of tarsal trauma and $2 \%$ of all fractures. The thalamic fractures constitute a particular entity by their mechanism of occurrence, by their treatment and by their prognosis much less favorable than the extra-articular fractures. ${ }^{1}$

Materials and methods: Our series spanning a period of 7 years from January 2011 to November 2018 focused on 12 patients with a thalamic fracture of the calcaneus treated by anatomical plate with bearing, collected in the Orthopedic Trauma Department A of the Hassan II Fez University Hospital. All adult patients with a thalamic calcaneal fracture who had undergone an osteosynthesis using an anatomical plate were included and extrathalamic fractures and thalamic fractures treated by other therapeutic means or other types of screw plates were excluded. All patients underwent an urgent general systematic examination and a regional examination. On the neurological level, the majority of patients were admitted with a GCS at 15 .
\end{abstract}

Results: Our focused on 12 operated patients between the ages of 17 and 53 with an average of 35 years. Our series includes 12 patients including 17 calacneum fractures which are divided into 10 men and 2 women; the male sex represents $83.3 \%$. The preoperative delay was on average 9.25 days with extremes between 03 and 20 days. No thromboembolic complications or algodystrophy were noted in our series. According to Kitaoka's rating, the average score was $73.5 \%$. The functional results were excellent in $47.06 \%$ of the cases, good in $23.53 \%$ of the cases, average in $23.53 \%$ of the cases and poor in $5.88 \%$ of the cases. In post-operative the average Bohler angle was $26.5^{\circ}\left(15^{\circ}\right.$ to $\left.35^{\circ}\right)$ against a pre-operative Bohler angle varying between $-7^{\circ}$ to $15^{\circ}$. On revision, the average recoil from Bohler's angle finds it to be $21.5^{\circ}$

Conclusion: In the light of all those preceding the surgical treatment of articular fractures of the calcaneus displaced by anatomical plate, despite the risks associated with this technique provides a certain superiority if you are sure you can get a perfect reduction.
Volume 12 Issue 3 - 2020

\author{
Lahrach K,' Soumare B,' Sidibe S,' Diarra S, \\ Saeed AR,' Boutayeb F' \\ 'Department of Traumatology and Orthopedics, Sidi \\ Mohammed Ben Abdellah University of Fez, Morocco \\ ${ }^{2}$ Laboratory of Epidemiology and Public Health, Sidi Mohammed \\ Ben Abdellah University of Fez, Morocco
}

\begin{abstract}
Correspondence: Soumaré Boubacar, Resident Doctor in Traumatology and Orthopedics at the Hassan II University Hospital, Faculty of Medicine and Pharmacy, Sidi Mohammed Ben Abdellah University of Fez, Morocco, Tel $00212628815726 / 22376371922$, Email docsoum9@gmail.com
\end{abstract}

Received: May 15, 2020 | Published: June 16, 2020

Keywords: thalamic fracture, anatomical plate, CHU Hassan II of Fez

\section{Introduction}

Calcaneus fractures are frequent and serious injuries. They represent $65 \%$ of tarsal trauma and $2 \%$ of all fractures. The thalamic fractures constitute a particular entity by their mechanism of occurrence, by their treatment and by their prognosis much less favorable than the extra-articular fractures. ${ }^{1}$ They are often the consequence of high energy accidents such as falling from a high place. ${ }^{2}$ The standard calcaneus X-ray with computed tomography (CT) allows good visualization of the lesions and guides the management. Surgical treatment is currently part of the therapeutic arsenal of calcaneal joint fractures, however the choice of the means of osteosynthesis in particular between osteosynthesis with closed hearth or with open hearth as well as the utility of the addition of a bone graft remain subject to discussion. Surgical treatment of displaced joint fractures of the calcaneus is recommended by most contemporary authors., ${ }^{3,4}$ Resumption of professional activities seems more constant and early after surgical treatment. ${ }^{5}$ The aim of our work is to evaluate in our department the results of the surgical treatment of thalamic fractures treated by anatomical plate with bearing and compare with data from the literature. Resumption of professional activities seems more constant and early after surgical treatment. ${ }^{5}$ The aim of our work is to evaluate in our department the results of the surgical treatment of thalamic fractures treated by anatomical plate with elevation and compare with data from the literature. Resumption of professional activities seems more constant and early after surgical treatment. ${ }^{5}$

\section{Materials and method}

Our series spanning a period of 7 years from January 2011 to November 2018 focused on 12 patients with a thalamic fracture of the calcaneus treated by anatomical plate with bearing, collected in the Orthopedic Trauma Department A of the Hassan II Fez University Hospital. All adult patients with a thalamic calcaneal fracture who had undergone an osteosynthesis using an anatomical plate were included and extra-thalamic fractures and thalamic fractures treated by other therapeutic means or other types of screw plates were excluded. All patients underwent an urgent general systematic examination and a regional examination. On the neurological level, the majority of patients were admitted with a GCS at 15 . All our patients benefited from a standard radiological assessment consisting at least of ankle radiography of the face, in profile and a retro-tibial radiograph and a CT scan. We used the classification of Duparc ${ }^{6}$ and the classification Uthéza $^{7}$ for their educational values allowing to better understanding the anatomo-pathology of thalamic fractures of the calcaneus. All of our patients were installed in strict lateral decubitus mainly under 
spinal anesthesia. A pneumatic tourniquet was placed at the root of the thigh. The incision was under and retromalleolar in L. an exposure by location of the sural nerve, the dissection is made without detachment until the periosteum; an arthromy and identification of the lateral peroneal muscles which will be raised as a whole (their sheath is respected); The reduction is carried out in several stages, its stages vary depending on the type of fracture. After checking the reduction under image intensification, settlement was carried out in most cases fixed by temporary pins perpendicular to the fundamental line. All our patients benefited from an osteosynthesis by special anatomical calcaneus plates in Y with different sizes. After a plan-by-plan closure, all our patients benefited from drainage (duration: 2 days) with the placement of a plastered splint in a boot. Post-operative: The Redon drain was removed on the 48th day; Get up from the 3rd4th day with canes-crutches, without support; Ablation of the sons on the 14th-15th day; Functional rehabilitation, including active and passive mobilization of the tibio-tarsal, of the mid-tarsus and the subtalar, Walking with canes-crutches with the only "foot-contact" support authorized around the 60th day, date on which full support is authorized. The patients were followed regularly in consultation. With an average decline of one year. Our results were evaluated according to Bohler's ${ }^{8}$ angle post-operatively and the functional score of kitaoka A radiological assessment was carried out every 3 months for a year.

\section{Results}

Our focused on 12 operated patients between the ages of 17 and 53 with an average of 35 years. Our series includes 12 patients including 17 calacneum fractures which are divided into 10 men and 2 women; the male sex represents $83.3 \%$. Falling from a high place: was the most common mechanism with 11 cases or $91.7 \%$ (The height ranges from 3 to 10 meters, 2 of which were due to a work accident). The right side was affected in 5 patients or $41.7 \%$, the left side was affected in 2 patients or $16.7 \%$ while bilateral involvement was found in 5 patients or $41.7 \%$. The preoperative delay was on average 9.25 days with extremes between 03 and 20 days. The majority of patients were admitted with a GCS at 15 with the presence of edema and bruising. The skin opening was noted in a single patient classified stage I of Cauchoix and Duparc and presence of abrasions in another patient. No vascular-nervous lesions were detected. In our series, 9 patients were admitted for polyfracture, or $75 \%$. The associated lesions were: Fracture of the contralateral calcaneus 5 cases; Tibial pestle + external malleolus 1 case; Astragalus fracture 1 case; Luxation of the ankle 1 case; Trauma to the spine 3 cases; Fracture of the lower extremity of the radius 2 cases; Scaphoid fracture 1 case; Trochanterodiaphyseal fracture of the femur 1 case. The fractures were type III Duparc in $23.53 \%$ of cases (4 patients), type IV in $29.41 \%$ ( 5 patients), and type $\mathrm{V}$ in $47.06 \%$ of cases (8 patients). The thalamic depression was horizontal in our series, 9 patients were admitted for polyfracture, or $75 \%$. The associated lesions were: Fracture of the contralateral calcaneus 5 cases; Tibial pestle + external malleolus 1 case; Astragalus fracture 1 case; Luxation of the ankle 1 case; Trauma to the spine 3 cases; Fracture of the lower extremity of the radius 2 cases; Scaphoid fracture 1 case; Trochantero-diaphyseal fracture of the femur 1 case. The fractures were type III Duparc in $23.53 \%$ of cases (4 patients), type IV in $29.41 \%$ (5 patients), and type $\mathrm{V}$ in $47.06 \%$ of cases ( 8 patients). The thalamic depression was horizontal in our series, 9 patients were admitted for polyfracture, or $75 \%$. The associated lesions were: Fracture of the contralateral calcaneus 5 cases; Tibial pestle + external malleolus 1 case; Astragalus fracture 1 case; Luxation of the ankle 1 case; Trauma to the spine 3 cases; Fracture of the lower extremity of the radius 2 cases; Scaphoid fracture 1 case; Trochantero-diaphyseal fracture of the femur 1 case. The fractures were type III Duparc in $23.53 \%$ of cases (4 patients), type IV in $29.41 \%$ (5 patients), and type V in $47.06 \%$ of cases ( 8 patients). The thalamic depression was horizontal in Luxation of the ankle 1 case; Trauma to the spine 3 cases; Fracture of the lower extremity of the radius 2 cases; Scaphoid fracture 1 case; Trochanterodiaphyseal fracture of the femur 1 case. The fractures were type III Duparc in $23.53 \%$ of cases (4 patients), type IV in $29.41 \%$ (5 patients), and type $\mathrm{V}$ in $47.06 \%$ of cases ( 8 patients). The thalamic depression was horizontal in Luxation of the ankle 1 case; Trauma to the spine 3 cases; Fracture of the lower extremity of the radius 2 cases; Scaphoid fracture 1 case; Trochantero-diaphyseal fracture of the femur 1 case. The fractures were type III Duparc in $23.53 \%$ of cases (4 patients), type IV in $29.41 \%$ (5 patients), and type V in $47.06 \%$ of cases ( 8 patients). The thalamic depression was horizontal in 2 sick, vertical at 3 patients and mixed in 12 patients. All our patients were installed in strict lateral decubitus. The intervention took place under spinal anesthesia in 12 cases $70.59 \%$ and under general anesthesia in 5 cases, i.e. $29.41 \%$. The incision was under and retro-malleolar extended in "L". Three patients in our series with a comminuted calcaneus fracture with thalamic depression had benefited from a bone graft or $17.65 \%$, two grafts by bone substitute were performed in patients with a type V Duparc fracture or $11.76 \%$. All our patients benefited from an osteosynthesis by special anatomical calcaneus plate in Y. The average duration of the intervention was 120 min with extremes ranging from 46 to $300 \mathrm{~min}$. The average length of hospital stay was 13 days with extremes of 6 days and 26 days. In our series, the complications listed were: - Superficial necrosis of the cutaneous banks early on D +5 and which healed later without repercussions: 1 case. - Joint stiffness: 2 case later corrected by rehabilitation. - Sepsis: 1 only case occurring at 1 year and which despite iterative trimming and removal of the osteosynthesis material, he presented after 2 years a significant loss of substance which required a skin graft. - Subtalar arthritis: 1 case no thromboembolic complications or algodystrophy were noted in our series. According to Kitaoka's rating, the average score was $73.5 \%$. The functional results were excellent in $47.06 \%$ of the cases, good in $23.53 \%$ of the cases, average in $23.53 \%$ of the cases and poor in $5.88 \%$ of the cases. In post-operative the average Bohler angle was $26.5^{\circ}$ $\left(15^{\circ}\right.$ to $\left.35^{\circ}\right)$ against a pre-operative Bohler angle varying between $-7^{\circ}$ to $15^{\circ}$. On revision, the average recoil from Bohler's angle finds it to be $21.5^{\circ}$. The average loss is evaluated at $2.5^{\circ}$. - Sepsis: 1 only case occurring at 1 year and which despite iterative trimming and removal of the osteosynthesis material, he presented after 2 years a significant loss of substance which required a skin graft. - Subtalar arthritis: 1 case no thromboembolic complications or algodystrophy were noted in our series. According to Kitaoka's rating, the average score was $73.5 \%$. The functional results were excellent in $47.06 \%$ of the cases, good in $23.53 \%$ of the cases, average in $23.53 \%$ of the cases and poor in $5.88 \%$ of the cases. In post-operative the average Bohler angle was $26.5^{\circ}\left(15^{\circ}\right.$ to $\left.35^{\circ}\right)$ against a pre-operative Bohler angle varying between $-7^{\circ}$ to $15^{\circ}$.

\section{Discussion}

Despite years of controversy, many authors currently agree on the need for surgical treatment in calcaneum joint fractures. ${ }^{1,10}$ In recent years, thanks to a better analysis of pathological lesions and the establishment of technical bases for the reduction and open osteosynthesis of calcaneal joint fractures, many authors have reported satisfactory results after the surgical treatment. ${ }^{11-13}$ Calcaneus fractures are possible at all ages, but there seems to be a peak in young age between 30 and 40 years old. This notion has been found in the 
literature as well as in our study. ${ }^{14,15}$ Falling from a high place remains the most common mechanism, as is the case in our series. We find in the literature according to Duparc and Caffinière a predominance of types III and IV (50\% and 70\%) compared to types I and II (10\% and $5 \%),{ }^{12,16-18}$ while in our work, there is a predominance of types $\mathrm{V}$ $(47.06 \%)$ and IV (29.41\%). According to the Utheza ${ }^{7}$ Classification the mixed form was in fact the most frequent form in our study $(70.59 \%)$ as in the Jellali ${ }^{16}$ series] with $60 \%$ on the other hand in the Nouissri ${ }^{17}$ series the vertical form was the most frequent with $79 \%$. We used the extended "L" lateral route in all our patients and From the literature series, it appears that all operators agree on the extended " $L$ " lateral route because it gives an excellent day on the lateral face of the calcaneus without cutaneous tension, makes it possible to visualize the entire calcaneus, as well as the subtalar and calcaneal-cuboid joints. ${ }^{15,18,19}$ The average correction of the Bolher angle in our series from $2.5^{\circ}$ in pre-op to $26.5^{\circ}$ in post-op is comparable to many series in the literature. ${ }^{16,17}$ At the one-year follow-up in our series, the average loss was evaluated at $2.5^{\circ}$, this value is comparable to secondary losses after osteosynthesis by plaque published by Thermann et al. ${ }^{20}$ Our functional results were comparable to the data in the literature with $70.59 \%$ of good to excellent results. ${ }^{21,} 22$ The most common complications according to the different series, not necessarily linked to the osteosynthesis techniques by plaque are: subtalar osteoarthritis, joint stiffness, algodystrophy, skin necrosis; even if their rates remain relatively low in our series with respectively, 1 case, 2 cases, 0 cases and 1 case. Subtalar osteoarthritis is the most formidable complication in the long run, even if this risk seems to be reduced following a plate osteosynthesis. ${ }^{23}$ Five of our patients have benefited from a transplant despite this attitude remains a subject of controversy in the literature. $^{24-26}$

\section{Conclusion}

In the light of all those preceding the surgical treatment of articular fractures of the calcaneus displaced by anatomical plate, despite the risks associated with this technique provides a certain superiority if you are sure you can get a perfect reduction.

\section{Acknowledgments}

None.

\section{Conflicts of interest}

No potential conflicts of interest relevant to this article have been reported.

\section{Funding}

None.

\section{References}

1. Juliano P, Nguyen HV. Fractures of the calcaneus. Orthop Clin North Am. 2001;32(1):35-51.

2. Goldzak M. Recent articular fractures of the calcaneus the observatory of movement. 2012.

3. Buckley R, Tough S, Mccormack R, et al. Operative compared with nonoperative treatment of displaced intraarticular calcaneal fractures. $J$ Bone Joint Surg Am. 2002;84(10):1733-1744.

4. Howard JL, Buckley R, Mccormack R, et al. Complications following management of displaced intra6articular calcaneal fractures: a prospective randomized trial comparing open reduction internal fixation with nonoperative management. J Orthop Trauma. 2003;17(4):241-249.
5. Paul M, Peter R, Hoffmeyer P. Fractures of the calcaneum. A review of 70 patients. J Bone Joint Surg Br. 2004;86(8):1142-1145.

6. Duparc J, Caffinière JY. Mechanism, anatomopathology, classification of articular fractures of the calcaneus. Ann Chir. 1970;24(5):289-301.

7. Uthéza G, Goldzak M, Chaminade B, et al. Three-dimensional imaging in thalamic fractures of the calcaneum. Validation of the classification in three forms. Rev Chir Orthop. 1998;84(5):440-450.

8. Bohler L. Diagnosis, Pathology and treatment of fractures of the os calcis. J Bone Joint Surg Am. 1931;13(1):75-89.

9. Kitaoka HB, Alexander IJ, Adelaar RS, et al. Clinical rating systems for the ankle-hindfoot, midfoot, hallux, and lesser toes. Foot Ankle Int. 1994;15(7):349-353.

10. Palmer I. The mechanism and treatment of fractures of the calcaneus. $J$ Bone Joint Surg Am. 1948;30A(1):2-8.

11. Buckley RE, Meek RN. Comparison of open versus closed reduction of intraarticular calcaneal fractures: a matched cohort in workmen. J Orthop Trauma. 1992;6(2):216-222.

12. Crosby LA, Fitzgibbons TC. Open reduction and internal fixation of type II intra-articular calcaneus fractures. Foot Ankle Int. 1996;17(5):253-258.

13. Parmar HV, Triffitt PD, Gregg PJ. Intra-articular fractures of the calcaneum treated operatively or conservatively: à prospective study. $J$ Bone Joint Surg Br. 1993;75 (6):932-937.

14. Bégué T, Mebtouche N, Auregan JC, et al. External fixation of the thalamic portion of a fractured calcaneus: A new surgical technique. Orthop Traumatol Surg Res. 2014;100(4):429-432.

15. Babin SR, Dossa J, Copin G. Thalamic fractures of the calcaneus and evolution in civil practice Symposium SOFCOT 1988. Rev chir orth. 1989;75(suppl 1):61-114.

16. Jellali T, Ouadih R, Zujevic J, et al. Treatment by plaque reconstruction of thalamic fractures of the calcaneus in 20 cases.

17. Nouissri L. Surgery of the calcaneus fractures of the paratrooper. International Review of Armed Forces Health Services. 2010:81-81.

18. Di Shino M, Bensaida M, Vandenbussche E, et al. Results of the treatment of calcaneus joint fractures by open hearth recovery and systematic grafting using the Palmer method. About 18cases. Rev Chir Orthop. 2008;94(2):135-144.

19. Freeman B, Duff S, Allene E, et al. The extended lateral approach to the hindfood. Anatomical basis and surgical implications. $J$ Bone Joint Surg (Br). 1998;80(1):139-142.

20. Thermann H, Krettek C, Hüfner T, et al. Management of calcaneal fractures in adults: Conservative versus operative treatment. Clin Orthop Relat Res. 1998;(353):107-124.

21. Schmidt TD, Mole D, Coudane H, et al. Conventional osteosynthesis in calcaneus fractures. Rev Chir Orthop. 1989;(75):83-84.

22. Bèzes H, Massart P, Delvaux D, et al. The Operative Treatment of Intraarticular Calcaneal Fractures Indications, Technique, and Results in 257 Cases. Clin Orthop Relat Res. 1993;(290):55-59.

23. Galvik JM, Rammelt S, Zwipp H. Percutaneus, arthroscopically-assised osteosynthesis of calcaneus fractures. Arch Orthop Trauma Surg. $2002 ; 122(8): 424-428$

24. Wilmoth P. Treatment of calcaneus fractures. $J$ Med Chir Prat. 1931;(102):328-335.

25. Geel CW, Flemister AS Jr. Standardized treatment of intra-articular calcaneal fractures using an oblique lateral incision and no bone graft. $J$ Trauma. 2001;50(6):1083-1089.

26. Letournel E. Open treatment of acute calcaneal fractures. Clin Orthop Relat Res. 1993;(290):60-67. 